\title{
Synergistic effect of halofuginone and dexamethasone on LPS-induced acute lung injury in type II alveolar epithelial cells and a rat model
}

\author{
HAI-LIAN DU ${ }^{1}$, AI-DONG $\mathrm{ZHAI}^{2}$ and HONG YU ${ }^{3}$ \\ ${ }^{1}$ Department of Respiratory Medicine, Yidu Central Hospital Affiliated to Weifang Medical College, Qingzhou, \\ Shandong 262500; ${ }^{2}$ Department of Internal Medicine, Maternal and Child Health Hospital of Zibo, Zibo, \\ Shandong 255029; ${ }^{3}$ Intensive Care Unit, Second Hospital of Harbin City, Harbin, Heilongjiang 150036, P.R. China
}

Received February 6, 2018; Accepted November 9, 2018

DOI: $10.3892 / \mathrm{mmr} .2019 .10865$

\begin{abstract}
Acute lung injury (ALI) is characterized by neutrophilic infiltration, uncontrolled oxidative stress and inflammatory processes. Despite various therapeutic regimes having been performed, there remains no effective pharmacotherapy available to treat ALI. Halofuginone (HF), a ketone isolated from Dichroa febrifuga, exhibits significant anti-inflammatory and antifibrotic effects. Dexamethasone (DEX), a synthetic glucocorticoid, has been routinely used as an adjuvant therapy in treating inflammatory diseases, including ALI. The present study aimed to investigate the effects of the combination of HF and DEX in the treatment of ALI. The present results suggested that the simultaneous administration of HF and DEX markedly decreased the level of pro-inflammatory cytokines and increased the level of anti-inflammatory cytokines, as assessed by western blot analysis. In addition, HF and DEX effectively decreased nuclear factor- $\kappa \mathrm{B}$ activity via suppressing the phosphorylation of P65 in lipopolysaccharide (LPS)-induced human pulmonary alveolar epithelial cells (HPAEpiC) and lung tissues extracted from ALI rats, as determined by immunofluorescence. Furthermore, in vivo experiments demonstrated that the combination of HF and DEX in LPS-induced ALI rats defended against lung fibrosis, perivascular inflammation, congestion and edema of pulmonary alveoli, as assessed by
\end{abstract}

Correspondence to: Dr Hong Yu, Intensive Care Unit, Second Hospital of Harbin City, 38 Weixing Road, Daowai, Harbin, Heilongjiang 150036, P.R. China

E-mail: yuhong0302@sina.com

Abbreviations: ALI, acute lung injury; HF, halofuginone; DEX, dexamethasone; LPS, lipopolysaccharide; HPAEpiC, type II alveolar epithelial cells; NF- $\kappa \mathrm{B}$, nuclear factor $\kappa \mathrm{B}$; TNF- $\alpha$, tumor necrosis factor- $\alpha$; IL-17, interleukin-17; IL-23, interleukin-23; IL-1 $\beta$, interleukin-1 $\beta$; SD rats, Sprague-Dawley rats

Key words: halofuginone, dexamethasone, acute lung injury, inflammatory cytokines, nuclear factor- $\mathrm{kB}$ pathway histopathological analysis, TUNEL staining and immunohistochemistry assay. Taken together, the present study indicated the synergistic effect of HF and DEX on LPS-induced ALI in HPAEpiC cells and a rat model. These results offer a novel therapeutic approach for the treatment of ALI.

\section{Introduction}

The histopathological features of acute lung injury (ALI) include alveolar leukocyte infiltration, lung edema, hyaline membrane formation, increased alveolar wall thickness and hemorrhage (1). A wide arrange of diseases can cause ALI, including pneumonia, sepsis and acute pancreatitis (2). Studies have shown that Gram-negative bacterial infection may be crucial in the development of ALI (3). Several natural products have been examined in experimental models and have been shown to inhibit multiple inflammatory pathways associated with ALI (4,5), however, the morbidity and mortality rates remain high (6). Therefore, the development of effective drugs or strategies to treat ALI is urgently required.

Halofuginone [HF, 7-bromo-6-chloro-3-[3-(3-hydroxy2-piperidinyl)-2-oxopropyl] is a low-molecular-weight plant-derived alkaloid extracted from Dichroa febrifuga. Previous studies have demonstrated that HF exhibits antifibrotic activity (7) and offers therapeutic promise in animal models of fibrotic disease (8). Other studies have indicated that HF has a beneficial effect in regulating the immune response (9). In addition, HF can reduce the productions of some pro-inflammatory cytokines, including tumor necrosis factor- $\alpha$ (TNF- $\alpha$ ) (10) and interleukin (IL)-17 (11), in inflammatory diseases.

Dexamethasone (DEX) is a potent, long-lasting synthetic glucocorticoid that possesses potent anti-inflammatory properties. According to previous reports, DEX can inactivate the nuclear factor $-\kappa \mathrm{B}(\mathrm{NF}-\kappa \mathrm{B})$ pathway (12) and inhibit the expression of IL-17 (13), IL-1 $\alpha$ (14) and IL-23 (15). For this reason, DEX is widely used to treat various inflammatory diseases, including rheumatoid arthritis (16), asthma (17) and ALI (18). According to previous reports, a combination of DEX with other drugs exerted superior effects on mitigating ALI $(19,20)$. Therefore, the present study aimed to examine the synergistic effect of DEX and HF in the treatment of ALI. 
The $\mathrm{NF}-\kappa \mathrm{B}$ pathway can activate an inflammatory cascade and lead to the upregulation of several pro-inflammatory cytokines, including TNF- $\alpha$ and IL-1 $\beta$ (21). According to published reports, the NF- $\kappa \mathrm{B}$ pathway is involved in the pathogenesis of ALI (22-24). In addition, during the pathological process of ALI, various pro-inflammatory cytokines are activated, particularly IL-17 (25) and IL-23 (26). This evidence demonstrates that NF- $\mathrm{BB}$ and the inflammatory cytokines are major targets for ALI therapy.

In the present study, the effect of DEX and HF on LPS-induced ALI and the underlying molecular mechanisms were examined in vitro and in vivo. Lipopolysaccharide (LPS)-induced type II alveolar epithelial cells (HPAEpiC cells) and ALI rats were treated with DEX, HF or their mixture respectively. The results demonstrated that there was a synergistic effect of HF and DEX on LPS-induced ALI in HPAEpiC cells and the rat model.

\section{Materials and methods}

Cell culture and treatment. The HPAEpiC human alveolar epithelial cells were purchased from ScienCell Company (Carlsbad, CA, USA; cat. no. 3200; https://www.sciencellonline.com/human-pulmonary-alveolar-epithelial-cells.html). The cells were cultured in DMEM (Gibco; Thermo Fisher Scientific, Inc., Waltham, MA, USA) supplemented with $10 \%$ fetal bovine serum (Gibco; Thermo Fisher Scientific, Inc.) at $37^{\circ} \mathrm{C}$ with $5 \% \mathrm{CO}_{2}$. The cells were divided into five groups: Control group: Normal HPAEPiC cells; LPS group: HPAEPiC cells incubated with $0.5 \mu \mathrm{g} / \mathrm{ml}$ LPS for $12 \mathrm{~h}$; LPS + DEX group: DEX (100 $\mathrm{nM})$ was administrated $12 \mathrm{~h}$ following LPS treatment; LPS + HF group: HF $(100 \mathrm{nM})$ was administrated $12 \mathrm{~h}$ following LPS treatment; LPS + DEX + HF group: HF $(100 \mathrm{nM})$ and DEX (100 $\mathrm{nM})$ mixture was administrated $12 \mathrm{~h}$ following LPS treatment. All cells were incubated at at $37^{\circ} \mathrm{C}$ with $5 \% \mathrm{CO}_{2}$.

Cell viability measurement. Cell viability was assessed using a 3-(4,5-dimethylthiazol-2-yl)-2,5-diphenyltetrazolium bromide (MTT; Sigma-Aldrich; Merck KGaA, Darmstadt, Germany) assay. The HPAEpiC cells were seeded into 96 -well plates at $5 \times 10^{3}$ cells/well and incubated in $5 \% \mathrm{CO}_{2}$ at $37^{\circ} \mathrm{C}$ for $12 \mathrm{~h}$. The culture medium was then replaced with fresh culture medium containing different doses of $\mathrm{HF}$ or DEX at the final concentration of $0,1,2,5,10,20,50,100,200$, and $400 \mathrm{nM}$. The surviving fractions were determined at 24 and $48 \mathrm{~h}$. Subsequently, the OD values were detected at $450 \mathrm{~nm}$ and normalized to cells treated with normal medium.

Western blot assay. The HPAEpiC cells subjected to different treatments for $24 \mathrm{~h}$ and the homogenized lung tissue samples were prepared for the western blot assay. All samples were lysed in RIPA lysis buffer containing $1 \%$ protease inhibitor. In total, $20 \mu \mathrm{g}$ protein was loaded in each lane. Proteins were separated by $12 \%$ SDS-PAGE and transferred onto PVDF (EMD Millipore, Billerica, MA, USA) membranes. The PVDF membranes were then blocked with $5 \%$ nonfat milk and washed with TBS with $5 \%$ Tween 20 at room temperature. Subsequently, the samples were incubated with anti-TNF- $\alpha$ [1:1,000; cat. no. 11948; Cell Signaling Technology, Inc. (CST)]; anti-IL-1 $\beta$
(1:1,000; cat. no. 12703 ; CST); anti-IL-17 (1:1,000; cat. no. 13838; CST); anti-IL-23 (1:100; cat. no. ab45420; Abcam); anti-IL-10 (1:1,000; cat. no. 12163; CST); anti-P65 (1:1,000; cat. no. 8242; CST) and anti-GAPDH (1:10,000; cat. no. ab181602; Abcam) primary antibodies overnight at $4^{\circ} \mathrm{C}$. Following extensive washing with TBST for three times, the membranes were incubated with horseradish peroxidase-labeled goat anti-rabbit secondary antibody (cat. no. ab6721; 1:2,000; Abcam) for 1.5 $\mathrm{h}$ at room temperature. The immunoreactive bands were visualized using a ChemiDoc XRS imaging system and Quantity One analysis software (version 1.42; Bio-Rad Laboratories, Inc., Franklin Lakes, NJ, USA).

$N F-\kappa B$ nuclear translocation. The HPAEpiC cells on a cover glass were washed with PBS and fixed in $4 \%$ paraformaldehyde. The fixed cells were then blocked with PBS containing $10 \%$ FBS for $30 \mathrm{~min}$ at room temperature. Subsequently, all samples were incubated with primary antibody P65 (1:200; cat. no. ab16502; Abcam) for $1 \mathrm{~h}$ at room temperature, followed by incubation with a secondary antibody conjugated with FITC (1:20,000; cat. no. ab6662; Abcam) for $30 \mathrm{~min}$ at room temperature. The samples were mounted in medium containing DAPI (Roche Diagnostics, Basel, Switzerland) for $5 \mathrm{~min}$ at room temperature. The location of $\mathrm{NF}-\kappa \mathrm{B}$ was measured using a laser scanning confocal microscope (magnification, x400; Olympus Corporation, Tokyo, Japan).

Animals and treatment. HF was purchased from Sigma-Aldrich (Merck KGaA). Specific-pathogen-free (SPF) Sprague-Dawley male rats (weight, 200-220 g; age, 7 weeks) were obtained from The Laboratory Animal Center of Yidu Central Hospital Affiliated to Weifang Medical College (Qingzhou, China). All rats were required to adapt to the environment for 7 days prior to the experiments with a maintained temperature of $22^{\circ} \mathrm{C}$ and a 12 -h light/dark cycle at $60 \%$ humidity. The animals were allowed free access to tap water and SPF fodder. The animal protocols used in the present study were approved by the Institutional Animal Ethics Committee and according to the Guidelines of Laboratory Animal Care and Use Committee. A total of 45 rats were randomly divided into five groups $(n=9$ per group). Control group: Normal rats; LPS group: Rats received an LPS ( $5 \mathrm{mg} / \mathrm{kg})$ intratracheal injection to induce ALI; LPS + DEX group: Rats were treated with DEX (5 mg/kg/d in PBS) $1 \mathrm{~h}$ following LPS treatment by intraperitoneal injection; LPS + HF group: Rats were treated with HF $(0.1 \mathrm{mg} / \mathrm{kg} / \mathrm{d}$ in PBS) $1 \mathrm{~h}$ following LPS treatment by intraperitoneal injection; LPS + DEX + HF group: HF $(15 \mathrm{mg} / \mathrm{kg} / \mathrm{d})$ and DEX $(0.1 \mathrm{mg} / \mathrm{kg} / \mathrm{d})$ was simultaneously administrated $1 \mathrm{~h}$ following LPS treatment. The treatment continued for 3 days. Subsequently, the rats were anesthetized with an intraperitoneal injection of pentobarbital sodium ( $30 \mathrm{mg} / \mathrm{kg}$ body weight). The lung tissues were collected from the rats under anesthesia for subsequent analyses. Following collection, the rats were sacrificed by intraperitoneal injection of pentobarbital sodium $(200 \mathrm{mg} / \mathrm{kg}$ body weight).

Histopathological analysis. The lung tissues were collected and washed with PBS following sacrifice of the rats. The tissues were then fixed with $10 \%$ paraformaldehyde and embedded in paraffin. The samples were cut into $4-\mu \mathrm{m}$ sections. Each 
A<smiles>O=C(C[C@@H]1NCCC[C@H]1O)Cn1cnc2cc(Br)c(Cl)cc2c1=O</smiles>
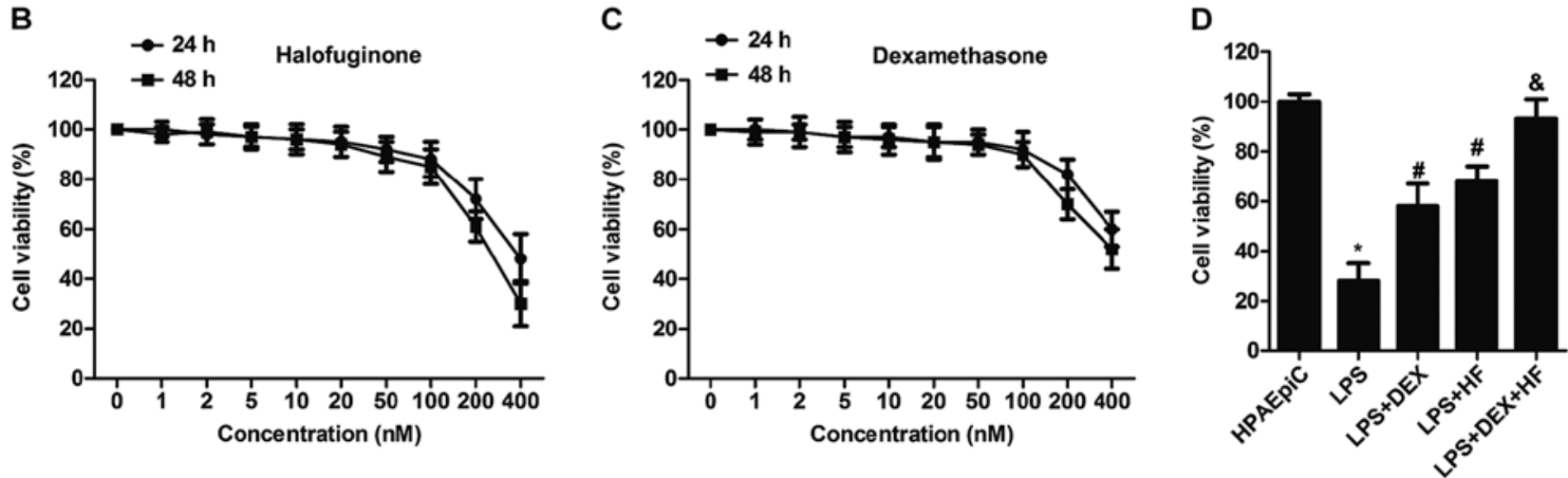

Figure 1. HF and DEX sustain the survival of HPAEpiC cells. (A) Structure of HF. (B) HPAEpiC cell viability was detected using an MTT assay. Cells were treated with $\mathrm{HF}$ at different concentrations $(0,1,2,5,10,20,50,100,200$, and $400 \mathrm{nM})$ for different durations (24 and $48 \mathrm{~h})$. (C) HPAEpiC cell viability was measured using an MTT assay. Cells were treated with DEX at different concentrations $(0,1,2,5,10,20,50,100,200$, and $400 \mathrm{nM})$ for different durations (24 and 48 h). (D) HPAEpiC cells were divided into five groups. Control group: Normal HPAEPiC cells; LPS group: HPAEPiC cells incubated with $0.5 \mu \mathrm{g} / \mathrm{ml}$ LPS for $12 \mathrm{~h}$; LPS + DEX group: DEX (100 nM) administrated $12 \mathrm{~h}$ following LPS treatment; LPS + HF group: HF (100 nM) administrated $12 \mathrm{~h}$ following LPS treatment; LPS + DEX + HF group: HF $(100 \mathrm{nM})+$ DEX $(100 \mathrm{nM})$ administrated $12 \mathrm{~h}$ following LPS treatment. Cell viability of HPAEpiC cells was measured using an MTT assay. The experiments were repeated at least three times $\left({ }^{*} \mathrm{P}<0.05\right.$ vs. HPAEpiC group; ${ }^{*} \mathrm{P}<0.05$ vs. LPS group; ${ }^{\&} \mathrm{P}<0.05$ vs. LPS+DEX group). HF, halofuginone; DEX, dexamethasone; LPS, lipopolysaccharide; MTT, 3-(4,5-dimethylthiazol-2-yl)-2,5-diphenyltetrazolium bromide.

section was stained with hematoxylin and eosin (H\&E). The histopathological features of ALI were assessed using light microscopy (magnification, x400; Olympus Corporation).

Terminal-deoxynucleotidyl transferase mediated nick end labeling (TUNEL) staining. According to the manufacturer's protocol, the lung tissue sections were fixed in acetone and slides were incubated with terminal deoxynucleotidyl transferase and detection buffer using the detection kit (Roche Molecular Biochemicals, Indianapolis, IN, USA). The numbers of TUNEL-positive nuclei were counted under a light microscope (magnification, $\mathrm{x} 400$; Olympus Corporation).

Immunohistochemistry assay for IL-17. The lung tissue sections underwent exposure to $3 \%$ hydrogen peroxide following dewaxing, hydration and antigen retrieval. The tissue sections were then incubated with anti-IL-17 primary antibodies (1:200; cat. no. ab79056; Abcam) overnight at $4^{\circ} \mathrm{C}$. The following day, biotin-labeled secondary antibody (1:250; cat. no. ab6658; Abcam) was added and incubated for $1 \mathrm{~h}$ at room temperature. The clay bank granules were observed under light microscopy.

Cytokine ELISA assay. The rats were anesthetized with an intraperitoneal injection of pentobarbital sodium $(30 \mathrm{mg} / \mathrm{kg}$ body weight) prior to cervical dislocation, following which blood was taken from the lung tissue sections. Following centrifugation at 3,000 $\mathrm{x}$ g for $15 \mathrm{~min}$ at $4^{\circ} \mathrm{C}$, serum was collected for the measurement of cytokine concentrations of IL-1 $\beta$ (Invitrogen; Thermo Fisher Scientific, Inc.), IL-23, TNF- $\alpha$ (PeproTech, Inc, Rocky Hill, NJ, USA), and IL-10 (eBioscience; Thermo Fisher Scientific, Inc.) using ELISA kits, following the manufacturer's protocol, at $450 \mathrm{~nm}$. Determinations were performed in duplicate in three independent experiments. The results are expressed as $\mathrm{pg} / \mathrm{ml}$.

Statistical analysis. Values are expressed as the mean \pm standard deviation. Comparisons between means were performed using one-way analysis of variance followed by Tukey's post hoc test using SPSS version17.0 (SPSS, Inc., Chicago, IL, USA). $\mathrm{P}<0.05$ was considered to indicate a statistically significant difference for all types of statistical test.

\section{Results}

HF and DEX sustain the survival of HPAEpiC cells. The structure of HF is illustrated in Fig. 1A. An MTT assay was used to determine the appropriate concentrations of HF and DEX. The HPAEpiC cell lines were treated with HF or DEX at different concentrations $(0,1,2,5,10,20,50,100,200$, and $400 \mathrm{nM}$ ), respectively. The results indicated that no significant decrease was observed in the HPAEpiC cells treated with HF or DEX concentrations $<100 \mathrm{~nm}$. However, cell viability was markedly reduced at a concentration $\geq 100 \mathrm{nM}$ (Fig. 1B and C). Therefore, the concentration of $100 \mathrm{nM}$ was selected for HF and DEX for the following experiments to exclude cell toxicity. The HPAEpiC cells were induced by LPS and treated with HF and DEX separately or together, the results showed that LPS reduced cell survival compared with the control group. However, the LPS-induced decrease in cell viability was elevated following treatment with HF or DEZ. Increased cell survival was observed in the $\mathrm{HF}+\mathrm{DEX}$ group compared with that in the DEX group (Fig. 1D).

HF and DEX weaken LPS-induced inflammatory response. To investigate the impact of HF and DEX on the inflammatory 
A

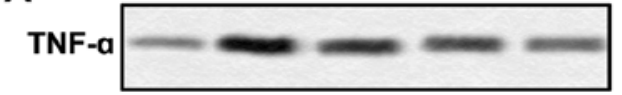

IL-1 $\beta$
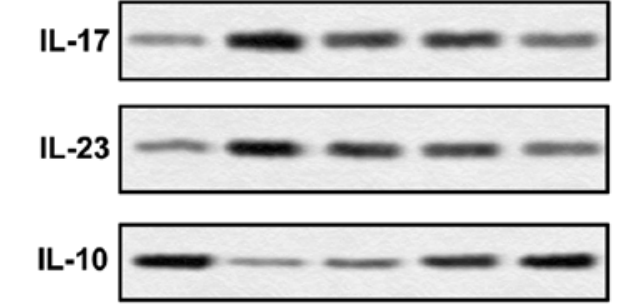

GAPDH

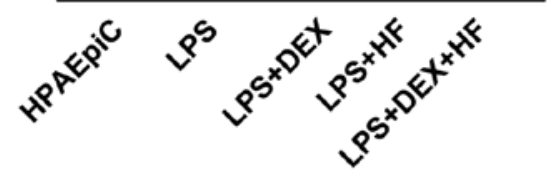

B

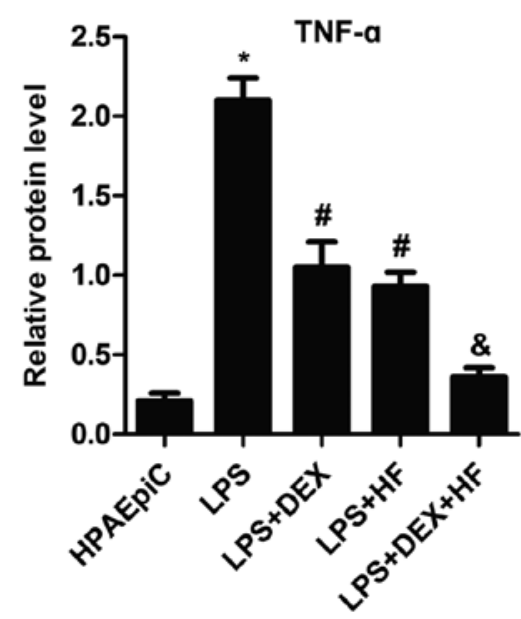

C

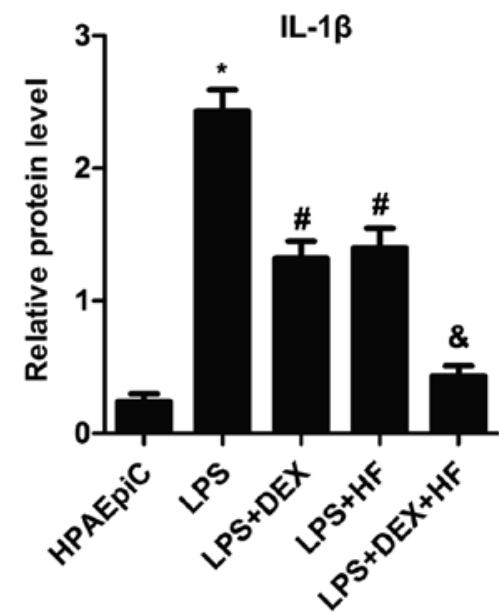

D

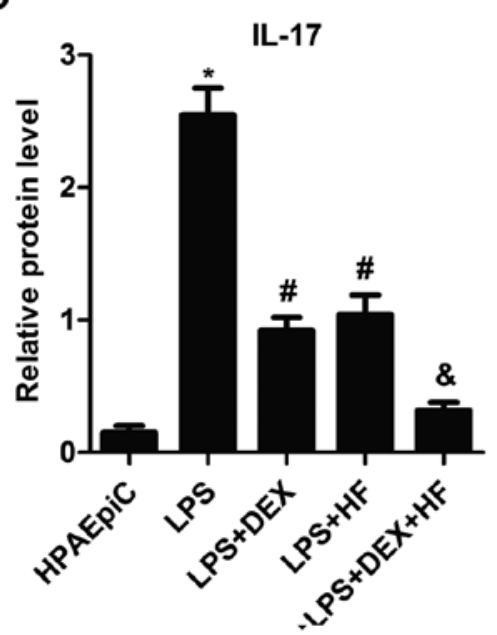

E

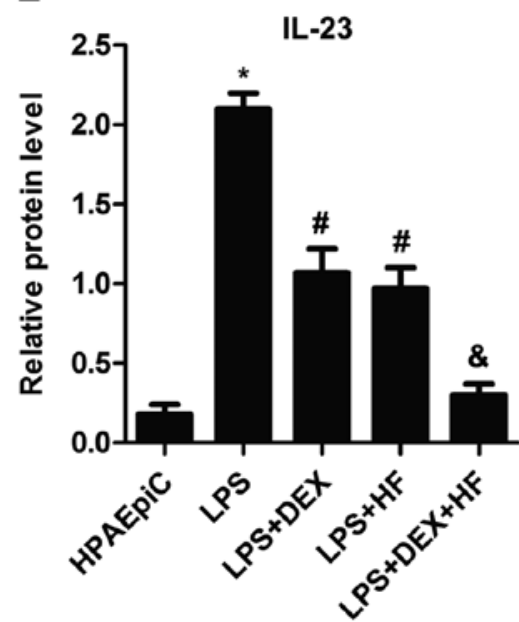

$\mathbf{F}$

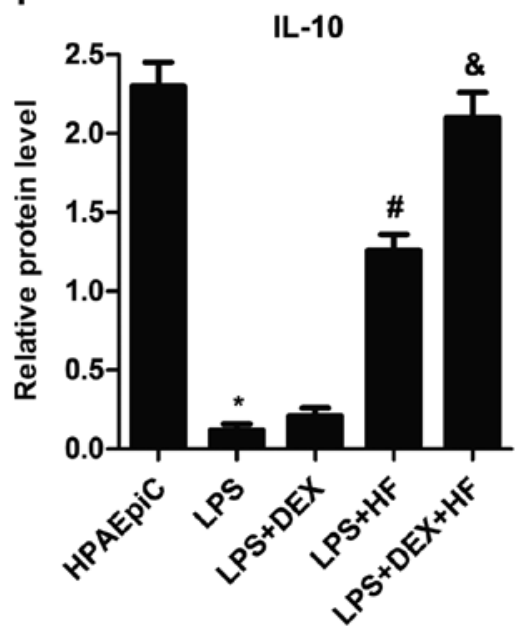

Figure 2. HF and DEX weaken the LPS-induced inflammatory response. (A) Expression levels of inflammatory cytokines (TNF- $\alpha$, IL-1 $\beta$, IL-17, IL-23 and IL-10) were detected using western blot analysis. GAPDH was the endogenous reference. Histograms showing the expression levels of (B) TNF- $\alpha$, (C) IL-1 $\beta$, (D) IL-17, (E) IL-23 and (F) IL-10 in HPAEpiC cells according to the results of western blot analysis. The experiments were repeated at least three times, and data are presented as the mean + standard deviation $\left({ }^{*} \mathrm{P}<0.05\right.$ vs. HPAEpiC group; ${ }^{\#} \mathrm{P}<0.05$ vs. LPS group; ${ }^{\star} \mathrm{P}<0.05$ vs. LPS+DEX group). HF, halofuginone; DEX, dexamethasone; LPS, lipopolysaccharide; TNF- $\alpha$, tumor necrosis factor- $\alpha$; IL, interleukin.

response, typical inflammatory cytokines were detected in the non-LPS or LPS-induced HPAEpiC cell lines. The western blot assays demonstrated that the expression levels of TNF- $\alpha$, IL-1 $\beta$, IL-17 and IL-23 were markedly increased, whereas that of IL-10 was markedly decreased, in the LPS-induced HPAEpiC cells compared with the normal HPAEpiC cells. In addition, the expression levels of TNF- $\alpha$, IL-1 $\beta$, IL-17, IL-23 were reduced, whereas that of IL-10 was elevated, in the LPS-induced cells treated with DEX or HF compared with the LPS-induced cells. Furthermore, the expression levels of TNF- $\alpha$, IL-1 $\beta$, IL-17 and IL-23 were suppressed, whereas that of IL-10 was upregulated, in the LPS-induced cells treated with DEX + HF, compared with those treated with DEX alone (Fig. 2A-F). These results indicated that the combination of HF and DEX can weaken the LPS-induced inflammatory response more than either HF or DEX used alone.
$H F$ and DEX reduce $N F-\kappa B$ activity via suppressing the phosphorylation of P65. To determine the mechanism underlying the effect of HF and DEX on the pro-inflammatory responses of HPAEpiC cells, the expression level of P65 and its phosphorylated form was measured by western blot analysis. As shown in Fig. 3A and B, the level of p-P65/P65 was upregulated in the LPS-induced HPAEpiC cells compared with that in the normal HPAEpiC cells. In addition, the expression of p-P65/P65 was reduced in the LPS-induced cells treated with DEX or HF, compared with that in the LPS group. Furthermore, the expression of p-P65/P65 was decreased in the LPS-induced cells treated with DEX $+\mathrm{HF}$ compared with those treated with DEX alone. To further validate this result, the subcellular localization of P65 was investigated using the immunofluorescence technique (Fig. 3C). The results indicated that the combination of DEX + HF decreased the nuclear translocation of $\mathrm{p} 65$. 
A
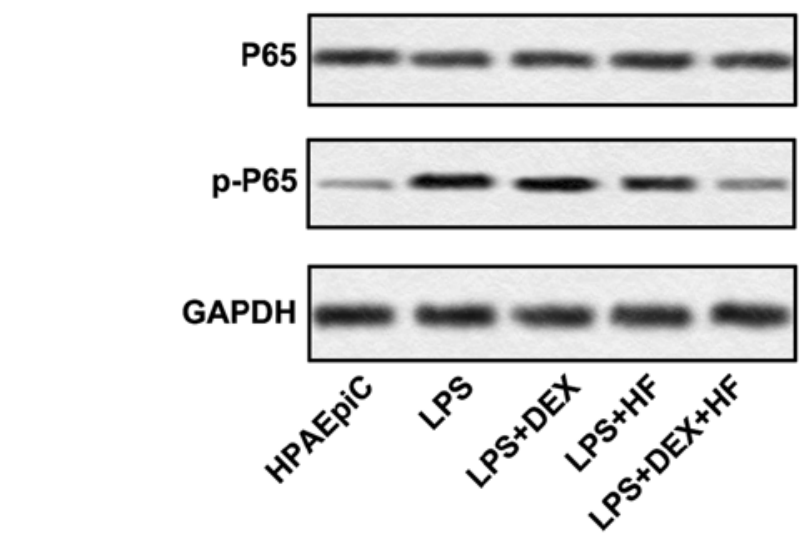

C
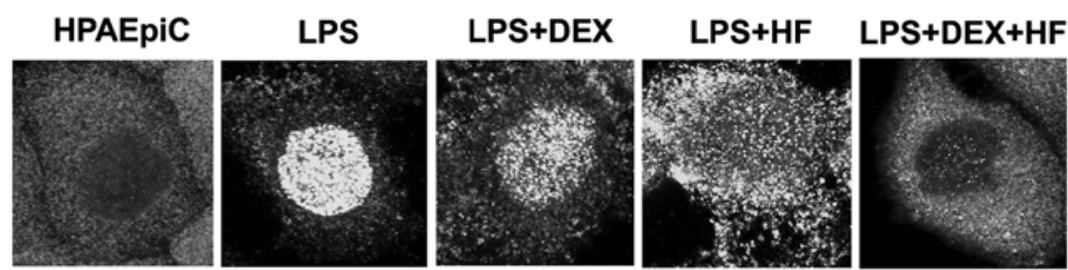

B
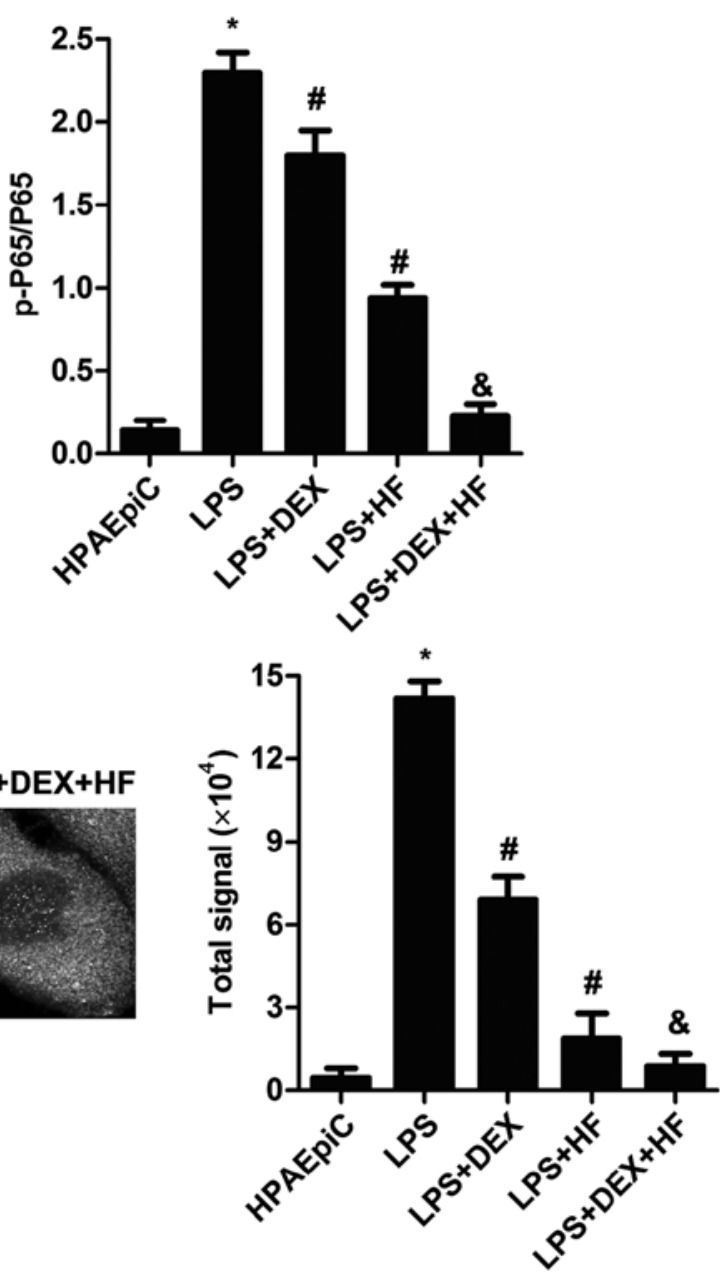

Figure 3. HF and DEX reduce the activity of NF-B via suppressing the phosphorylation of P65. (A) Expression levels of P65 and p-P65 were measured by western blot analysis. GAPDH was the endogenous reference. (B) Data summary and analysis of the expression of p-P65/P65 in cells according to the results of western blot analysis. (C) Nuclear translocation of p65 was measured by immunofluorescence (magnification, x400). The experiments were repeated at least three times, and data are presented as the mean + standard deviation ( ${ }^{*} \mathrm{P}<0.05$ vs. HPAEpiC group; $\mathrm{P}<0.05$ vs. LPS group; ${ }^{\text {}} \mathrm{P}<0.05$ vs. LPS+DEX group). $\mathrm{HF}$, halofuginone; DEX, dexamethasone; LPS, lipopolysaccharide; p-, phosphorylated.

HF and DEX alleviate acute lung injury in an LPS-induced rat model. To observe the impact of HF and DEX on morphological changes of lung tissues, H\&E staining was used. The results showed that, in the rats suffering from LPS-induced ALI with no treatment, minimal typical histomorphology of the lung was observed. By contrast, in the LPS-induced ALI rats treated with $\mathrm{HF}+\mathrm{DEX}$, the degree of perivascular inflammation and neutrophil infiltration was significantly decreased, compared with that in the LPS-induced ALI rats treated with DEX or HF alone. Furthermore, the combination of the two drugs reduced the congestion and edema of pulmonary alveoli, thickness of the pulmonary septum and fibrosis compared with the LPS-induced ALI rats treated with DEX or HF alone (Fig. 4A). Apoptotic bodies in the lung tissue were examined using a TUNEL assay. As shown in Fig. 4B and C, the numbers of apoptotic bodies were increased in the LPS-induced lung tissues compared with those in the normal lung tissues. In addition, apoptotic numbers were reduced in the LPS-induced lung tissues treated with DEX or HF compared with those in LPS-induced lung tissues. Furthermore, the numbers of apoptotic bodies were decreased in LPS-induced lung tissues treated with DEX + HF compared with those in the LPS-induced lung tissues treated with DEX alone (Fig. 4B and C). To confirm the effect of DEX and HF on inflammatory factors in LPS-induced lung tissues, immunohistochemical staining was used to investigate the expression of IL-17. The results suggested that the expression of IL-17 was markedly upregulated in the LPS-induced lung tissues compared with that in normal lung tissues. In addition, the expression of IL-17 was reduced in the LPS-induced lung tissues treated with HF or DEX compared with that in LPS-induced lung tissues. The expression level of IL-17 was decreased in the LPS-induced lung tissues treated with $\mathrm{HF}+\mathrm{DEX}$ compared with that in LPS-induced lung tissues treated with DEX alone (Fig. 4D and E). To identify whether DEX and HF activated the NF- $\kappa$ B pathway, western blot analysis was performed to measure the expression of P65 and its phosphorylated form in the lung tissues. The results demonstrated that the expression of p-P65/P65 was markedly increased in LPS-induced lung tissues compared with that in normal lung tissues. In addition, the expression of p-P65/P65 was reduced in the LPS-induced lung tissues treated with HF or DEX compared with that in LPS-induced lung tissues. Furthermore, the expression level of p-P65/P65 was decreased in the LPS-induced lung tissues treated with HF + DEX 

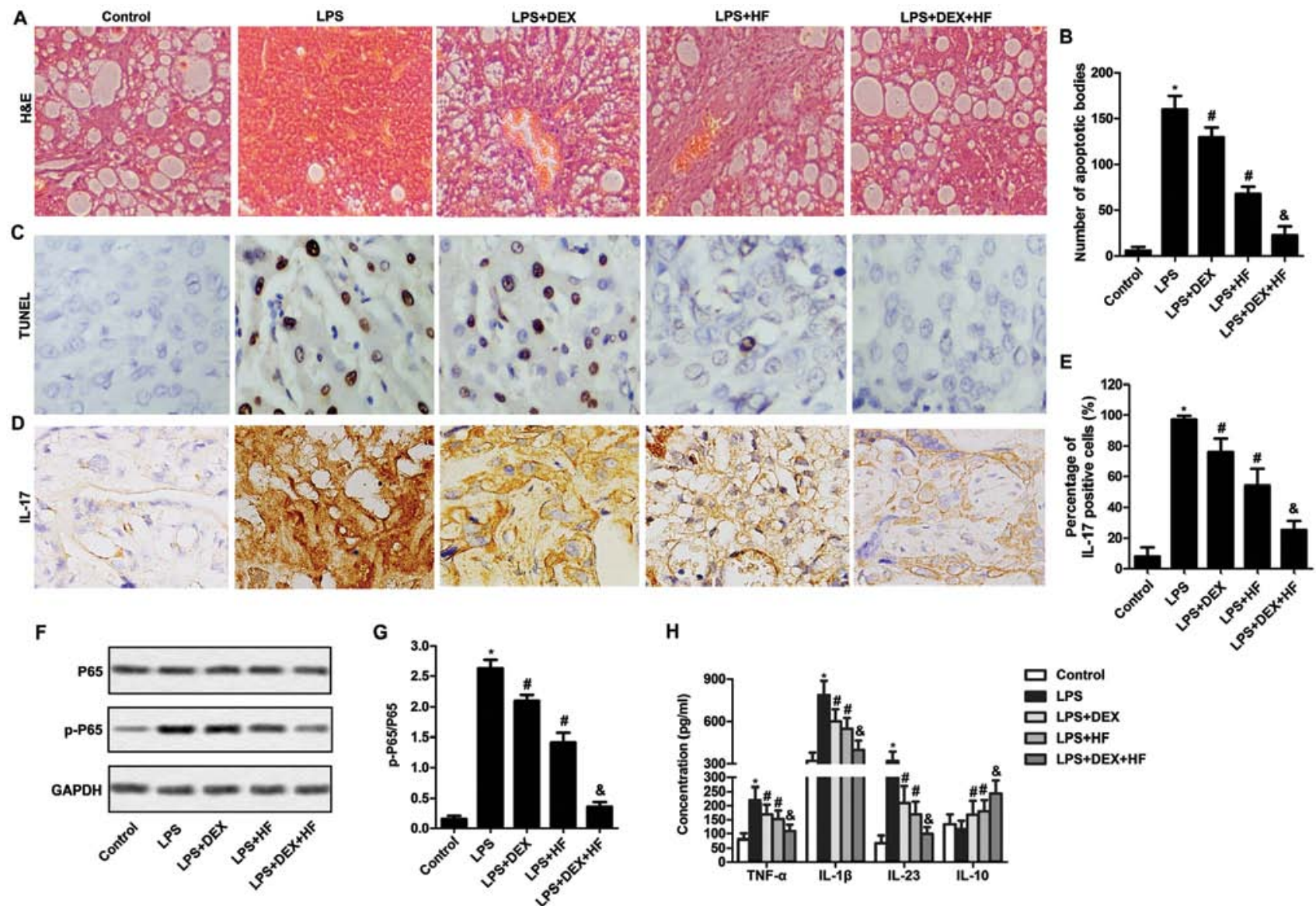

H

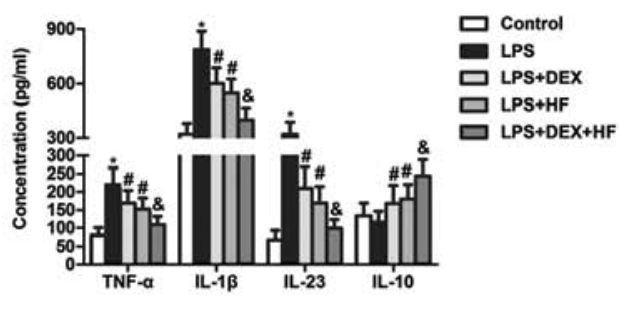

Figure 4. HF and DEX alleviate acute lung injury in an LPS-induced rat model. (A) H\&E staining to examine the morphological changes of lung tissues (magnification, x400). (B) Data summary and analysis of the number of apoptotic bodies according to the results of the TUNEL assay. (C) TUNEL assay used to detect the number of apoptotic bodies (magnification, x400). (D) Expression of IL-17 was observed through an immunohistochemical assay. Magnification, x400. (E) Histogram represents the percentage of IL-17-positive cells in lung tissue according to the results of immunohistochemical assay. (F) Expression levels of P65 and p-P65 were evaluated through western blot analysis. GAPDH was used as an endogenous reference. (G) Histogram showing the expression of p-P65/P65according to the results of western blot analysis. (H) Concentrations of TNF- $\alpha$, IL-1 $\beta$, IL-23 and IL-10 were measured using an ELISA assay. The experiments were repeated at least three times and data are presented as the mean + standard deviation $\left(" \mathrm{P}<0.05\right.$ vs. healthy control group; ${ }^{\prime \prime} \mathrm{P}<0.05$ vs. LPS group; ${ }^{\&} \mathrm{P}<0.05$ vs. LPS+DEX group). HF, halofuginone; DEX, dexamethasone; LPS, lipopolysaccharide; IL-17, interleukin-17; TNF- $\alpha$, tumor necrosis factor- $\alpha$; p-, phosphorylated; H\&E, hematoxylin and eosin; TUNEL, terminal-deoxynucleotidyl transferase mediated nick end labeling.

compared with that in LPS-induced lung tissues treated with DEX alone (Fig. 4F and G). Finally, the LPS-induced elevated levels of TNF- $\alpha$, IL-1 $\beta$ and IL-23 were decreased in the DEXor HF-treated cells. The decreased levels of TNF- $\alpha$, IL- $1 \beta$ and IL-23 and increased level of IL-10 were measured in the DEX + HF group and compared with those in the cells treated with DEX alone (Fig. 4H). These results suggested that the combination of HF and DEX led to further alleviation of ALI in the LPS-induced rat model.

\section{Discussion}

ALI is a clinical syndrome of severe lung failure. The complications of ALI include persistent respiratory failure, prolonged dependence on mechanical ventilation, multi-organ dysfunction and mortality (27). Despite progress having been made in drug exploration and understanding mechanisms, effective pharmacotherapy for ALI remains limited. Until now, a number of studies have demonstrated that several extracts from Chinese herbs can be of importance in treating ALI, including Sarcandra glabra (28), wogonin (29), flos lonicerae japonicae (30) and triptolide (31). These reports indicate the potential of Chinese herbs in treating ALI.

HF, a nontoxic antiparasitic alkaloid derivative of Dichroa febrifuga roots, has been widely used in the treatment of multiple inflammatory diseases, including colitis (32), autoimmune arthritis (9) and acute viral myocarditis (33). However, the use of HF to treat ALI has not been reported. DEX, a well-known anti-inflammatory agent, is also widely applied to treat various inflammatory diseases. According to published reports, DEX has been used to treat inflammatory bowel disease (34), inflammatory airway disease (35), corneal inflammation (36) and ALI (37). These studies suggest that the combination of HF and DEX is a promising therapeutic regime for the treatment of ALI.

Accumulated evidence has demonstrated that HF can affect various inflammatory cytokines. For example, the administration of HF has been shown to result in marked decreases in the levels of pro-inflammatory factors, including IL-6, TNF- $\alpha$ and IL-1 $\beta$ (38). In addition, it has been reported that HF treatment decreased IL-17A and improved features of chronic lung allograft dysfunction in a mouse orthotopic lung transplant 
model (11). According to Carlson et al (39), pro-inflammatory IL-23 responses can be selectively repressed by activation of the HF-induced amino acid starvation response in mature Th17 memory cells. DEX has been reported to affect multiple inflammatory cytokines to reduce inflammatory responses. It has been shown that stimulation of DEX with $\beta$-glucans markedly increased the secretion of IL-10 and phosphorylation of Syk, and decreased the production of IL-12, IL-23 and TNF- $\alpha$ (40). Furthermore, DEX was reported to suppress the serum level of IL-17 in a bleomycin A5-induced rat model of pulmonary fibrosis (13). Similarly, in the present study, HF or DEX used alone reduced the expression of pro-inflammatory cytokines (TNF- $\alpha$, IL-1 $\beta$, IL-17 and IL-23) and elevated the expression of the anti-inflammatory IL-10 cytokine. The combination of these two drugs led to a more marked decrease in pro-inflammatory cytokine and increase in anti-inflammatory cytokine expression.

The NF- $\kappa$ B signaling pathway is a crucial pathway which has been reported to be involved in the regulation of pro-inflammatory mediator generation (41). A number of studies have indicated that the $\mathrm{NF}-\kappa \mathrm{B}$ signaling pathway is activated in ALI $(22,23,42)$. In previous studies, DEX effectively decreased the activity of NF- $\mathrm{B}$ P65 $(40,43)$. In addition, increasing evidence suggests that various monomers of Chinese herbs can inactivate the NF- $\kappa \mathrm{B}$ signaling pathway to prevent the development of inflammatory diseases. According to $\mathrm{Xu}$ et al (44), $\beta$-glucan-induced macrophage activation can be suppressed by tetrandrine through inhibiting signal transducer and activator of transcription 3, extracellular signal-regulated kinase and $\mathrm{NF}-\kappa \mathrm{B}$ signaling pathways. Others have reported that the activation of $\mathrm{NF}-\kappa \mathrm{B}$ can be reduced by shikonin, leading to inhibited oxidized low-density lipoprotein-induced monocyte adhesion in EA.hy926 endothelial cells (45). A similar result was obtained in the present study; treatment with DEX or HF alone reduced the activity of $\mathrm{NF}-\kappa \mathrm{B}$ via suppressing the phosphorylation of P65. However, the simultaneous application of these two drugs decreased the phosphorylation of P65 more markedly than that with HF or DEX alone. In addition, other signaling pathways have been associated with LPS-induced lung injury. A study by Li et al (46) demonstrated that apigenin C-glycosides inhibit acute inflammation and apoptosis by suppressing activation of the toll-like receptor $4 /$ transient receptor potential cation channel, subfamily $\mathrm{C}$, member 6 signaling pathway in a murine model of ALI. Cordycepin exerted a protective effect on injuries in lung tissues via the nuclear factor, erythroid 2 like 2/heme oxygenase-1 pathway (47). The p38 mitogen-activated protein kinase signaling pathway was also shown to be involved in the LPS-induced excessive inflammatory responses in ALI (48). These pathways are to be examined in subsequent investigations to examine the compounds effects.

Progressive fibroproliferative diseases include liver cirrhosis, kidney fibrosis and pulmonary fibrosis. Pulmonary fibrosis is a chronic, incurable clinical disease, the therapeutic options for which are usually of limited success. Until now, an increasing number of studies have demonstrated that $\mathrm{HF}$ is vital in treating fibroproliferative diseases. According to Liang et al (38), the oral administration of HF had a potent effect against liver fibrosis by decreasing inflammation-mediated liver damage and the deposition of collagen I. In addition, it has been shown that esophageal and hypopharyngeal fibrosis can be safely prevented by HF (49). Nagler et al (50) observed that HF was a potent inhibitor of bleomycin-induced pulmonary fibrosis in vivo, and was suitable for use as a novel therapeutic regimen for the treatment of this dysfunction. In addition to this, DEX has also been reported to exhibit its protective effect in certain fibroproliferative diseases. Wang et al stated briefly that DEX defended against bleomycin A5-induced pulmonary fibrosis through decreasing the level of IL-17 in the serum of rats (13). A similar result was obtained in the present study; HF used alone suppressed lung fibrosis and the combination of DEX with HF enhance the curative effect.

The ALI model can be induced by different reagents; a rodent model of ALI can be induced by sulfur dioxide with DEX to evaluate whether the inflammatory response and lung fibrosis can be counteracted (51). According to a report by $\mathrm{Xu}$ et al (52), an ALI model was induced by oleic acid to measure the cell apoptotic pathway. The ALI model in the present study was established by intratracheal injection of LPS $(5 \mathrm{mg} / \mathrm{kg}$ ) in rats. According to a previous report, the intratracheal instillation of LPS induced a robust pulmonary pro-inflammatory response with endothelial barrier dysfunction (53). Dong et al (54) also used a rat model of ALI induced by LPS and an LPS-induced cell model to investigate the effect of carbon monoxide on ALI. As the present study focused on the role of immune adjustment by HF and DEX, LPS was selected to induce the ALI model. LPS-induced ALI has also been associated with sepsis and acute respiratory distress syndrome (55) in addition to the cecal ligation and puncture model of sepsis (56). The synergistic effect of HF and DEX on sepsis is to be investigated in our subsequent investigations.

Based on the experimental results, the present study suggested that HF enhanced the effect of DEX in sustaining survival and LPS-induced inflammatory response of HPAEpiC cells. The mechanism may be associated with inhibition of the phosphorylation of $\mathrm{NF}-\kappa \mathrm{B}$. In addition, HF may assist DEX in alleviating cell apoptosis and inflammatory responses in the LPS-induced rat model. These findings progress current understanding of the NFKB pathway associated with ALI elicited by the combination of DEX and HF.

\section{Acknowledgements}

The authors would like to thank Dr Feng Luan, Dr Yong Xu, Dr Jingwei Wang and Dr Ling Lu of Yidu Central Hospital Affiliated to Weifang Medical College (Qingzhou, China), for providing helpful discussions and technical support for the present study.

\section{Funding}

No funding was received.

\section{Availability of data and materials}

The datasets used and/or analyzed in the present study are available from the corresponding author on reasonable request. 


\section{Authors' contributions}

HLD analyzed and interpreted the main data regarding cell viability and ALI model establishment. ADZ was responsible for pathological and statistical analyses. HY was responsible for the design and drafting of the manuscript. All authors read and approved the final manuscript.

\section{Ethics approval and consent to participate}

The animal experiments in the present study were approved by the Animal Care and Research Committee of Yidu Central Hospital Affiliated to Weifang Medical College (Qingzhou, China). All experiments were performed in compliance with relevant laws and guidelines. All experiments were conducted according to the institutional guidelines of Yidu Central Hospital Affiliated to Weifang Medical College.

\section{Patient consent for publication}

Not applicable.

\section{Competing interests}

The authors declare that they have no competing interests.

\section{References}

1. Miyashita T, Ahmed AK, Nakanuma S, Okamoto K, Sakai S, Kinoshita J, Makino I, Nakamura K, Hayashi H, Oyama K, et al: A three-phase approach for the early identification of acute lung injury induced by severe sepsis. In Vivo 30: 341-349, 2016.

2. Zimmerman JJ, Akhtar SR, Caldwell E and Rubenfeld GD: Incidence and outcomes of pediatric acute lung injury. Pediatrics 124: 87-95, 2009.

3. Sawa T: The molecular mechanism of acute lung injury caused by Pseudomonas aeruginosa: From bacterial pathogenesis to host response. J Intensive Care 2: 10, 2014.

4. Hu Y, Ren J, Wang L, Zhao X, Zhang M, Shimizu K and Zhang C: Protective effects of total alkaloids from Dendrobium crepidatum against LPS-induced acute lung injury in mice and its chemical components. Phytochemistry 149: 12-23, 2018.

5. Patel VJ, Biswas Roy S, Mehta HJ, Joo M and Sadikot RT: Alternative and natural therapies for acute lung injury and acute respiratory distress syndrome. Biomed Res Int 2018: 2476824, 2018

6. Johnson ER and Matthay MA: Acute lung injury: Epidemiology, pathogenesis, and treatment. J Aerosol Med Pulm Drug Deliv 23: 243-252, 2010.

7. Dent P, Yacoub A, Contessa J, Caron R, Amorino G, Valerie K, Hagan MP, Grant S and Schmidt-Ullrich R: Stress and radiation-induced activation of multiple intracellular signaling pathways. Radiat Res 159: 283-300, 2003.

8. Pines M, Snyder D, Yarkoni S and Nagler A: Halofuginone to treat fibrosis in chronic graft-versus-host disease and scleroderma. Biol Blood Marrow Transplant 9: 417-425, 2003.

9. Park MK, Park JS, Park EM, Lim MA, Kim SM, Lee DG, Baek SY, Yang EJ, Woo JW, Lee J, et al: Halofuginone ameliorates autoimmune arthritis in mice by regulating the balance between Th17 and Treg cells and inhibiting osteoclastogenesis. Arthritis Rheumatol 66: 1195-1207, 2014.

10. Zeng S, Wang K, Huang M, Qiu Q, Xiao Y, Shi M, Zou Y, Yang X, $\mathrm{Xu} \mathrm{H}$ and Liang L: Halofuginone inhibits TNF- $\alpha$-induced the migration and proliferation of fibroblast-like synoviocytes from rheumatoid arthritis patients. Int Immunopharmacol 43: 187-194, 2017.

11. Oishi H, Martinu T, Sato M, Matsuda Y, Hirayama S, Juvet SC Guan Z, Saito T, Cypel M, Hwang DM, et al: Halofuginone treatment reduces interleukin-17A and ameliorates features of chronic lung allograft dysfunction in a mouse orthotopic lung transplant model. J Heart Lung Transplant 35: 518-527, 2016.
12. Gao W, Tong D, Li Q, Huang P and Zhang F: Dexamethasone promotes regeneration of crushed inferior alveolar nerve by inhibiting NF- $\kappa \mathrm{B}$ activation in adult rats. Arch Oral Biol 80: 101-109, 2017.

13. Wang A, Wang F, Yin Y, Zhang M and Chen P: Dexamethasone reduces serum level of IL-17 in Bleomycin-A5-induced rats model of pulmonary fibrosis. Artif Cells Nanomed Biotechnol 46: 783-787, 2018.

14. Roach BL, Kelmendi-Doko A, Balutis EC, Marra KG, Ateshian GA and Hung CT: Dexamethasone release from within engineered cartilage as a chondroprotective strategy against interleukin-1 $\alpha$. Tissue Eng Part A 22: 621-632, 2016.

15. Palma L, Sfara C, Antonelli A and Magnani M: Dexamethasone restrains ongoing expression of interleukin-23p19 in peripheral blood-derived human macrophages. BMC Pharmacol 11: 8, 2011.

16. Wang Q, Jiang H, Li Y, Chen W, Li H, Peng K, Zhang Z and Sun X: Targeting NF- $\mathrm{B}$ signaling with polymeric hybrid micelles that co-deliver siRNA and dexamethasone for arthritis therapy. Biomaterials 122: 10-22, 2017.

17. Shaughnessy AF: Single-dose dexamethasone an option for acute adult asthma. Am Fam Physician 95: Online, 2017.

18. Kosutova P, Mikolka P, Balentova S, Adamkov M, Kolomaznik M, Calkovska A and Mokra D: Intravenous dexamethasone attenuated inflammation and influenced apoptosis of lung cells in an experimental model of acute lung injury. Physiol Res 65 (Suppl 5): S663-S672, 2016.

19. Liu F, Pauluhn J, Trubel H and Wang C: Single high-dose dexamethasone and sodium salicylate failed to attenuate phosgene-induced acute lung injury in rats. Toxicology 315: 17-23, 2014.

20. Zhang S, Chen X, Devshilt I, Yun Q, Huang C, An L, Dorjbat S and He X: Fennel main constituent, trans-anethole treatment against LPS-induced acute lung injury by regulation of Th17/Treg function. Mol Med Rep 18: 1369-1376, 2018.

21. Park GY and Christman JW: Nuclear factor kappa B is a promising therapeutic target in inflammatory lung disease. Curr Drug Targets 7: 661-668, 2006.

22. Ho YC, Lee SS, Yang ML, Huang-Liu R, Lee CY, Li YC and Kuan YH: Zerumbone reduced the inflammatory response of acute lung injury in endotoxin-treated mice via Akt-NFKB pathway. Chem Biol Interact 271: 9-14, 2017.

23. Wang T, Hou W and Fu Z: Preventative effect of OMZ-SPT on lipopolysaccharide-induced acute lung injury and inflammation via nuclear factor-kappa B signaling in mice. Biochem Biophys Res Commun 485: 284-289, 2017.

24. Li N, Song Y, Zhao W, Han T, Lin S, Ramirez O and Liang L: Small interfering RNA targeting NF- $\mathrm{kB}$ attenuates lipopolysaccharide-induced acute lung injury in rats. BMC Physiol 16: 7, 2016.

25. Li Q, Gu Y, Tu Q, Wang K, Gu X and Ren T: Blockade of Interleukin-17 restrains the development of acute lung injury. Scand J Immunol 83: 203-211, 2016.

26. Yan B, Chen F, Xu L, Xing J and Wang X: HMGB1-TLR4-IL23-IL17A axis promotes paraquat-induced acute lung injury by mediating neutrophil infiltration in mice. Sci Rep 7: 597, 2017.

27. D'Alessio FR, Tsushima K, Aggarwal NR, West EE, Willett MH, Britos MF, Pipeling MR, Brower RG, Tuder RM, McDyer JF and King LS: CD4+CD25+Foxp3+ Tregs resolve experimental lung injury in mice and are present in humans with acute lung injury. J Clin Invest 119: 2898-2913, 2009.

28. Liu TY and Chen SB: Sarcandra glabra combined with lycopene protect rats from lipopolysaccharide induced acute lung injury via reducing inflammatory response. Biomed Pharmacother 84: 34-41, 2016.

29. Wei CY, Sun HL, Yang ML, Yang CP, Chen LY, Li YC, Lee CY and Kuan YH: Protective effect of wogonin on endotoxin-induced acute lung injury via reduction of p38 MAPK and JNK phosphorylation. Environ Toxicol 32: 397-403, 2017.

30. Kao ST, Liu CJ and Yeh CC: Protective and immunomodulatory effect of flos Lonicerae japonicae by augmenting IL-10 expression in a murine model of acute lung inflammation. J Ethnopharmacol 168: 108-115, 2015.

31. Wang X, Zhang L, Duan W, Liu B, Gong P, Ding Y and Wu X: Anti-inflammatory effects of triptolide by inhibiting the NF- $\kappa \mathrm{B}$ signalling pathway in LPS-induced acute lung injury in a murine model. Mol Med Rep 10: 447-452, 2014.

32. Liu J, Xiao HT, Wang HS, Mu HX, Zhao L, Du J, Yang D, Wang D, Bian ZX and Lin SH: Halofuginone reduces the inflammatory responses of DSS-induced colitis through metabolic reprogramming. Mol Biosyst 12: 2296-2303, 2016. 
33. Sun $\mathrm{XH}, \mathrm{Fu} J$ and Sun DQ: Halofuginone alleviates acute viral myocarditis in suckling BALB/c mice by inhibiting TGF- $\beta 1$. Biochem Biophys Res Commun 473: 558-564, 2016.

34. Dianzani C, Foglietta F, Ferrara B, Rosa AC, Muntoni E, Gasco P, Della Pepa C, Canaparo R and Serpe L: Solid lipid nanoparticles delivering anti-inflammatory drugs to treat inflammatory bowel disease: Effects in an in vivo model. World J Gastroenterol 23: 4200-4210, 2017.

35. Leguillette R, Tohver T, Bond SL, Nicol JA and McDonald KJ: Effect of dexamethasone and fluticasone on airway hyperresponsiveness in horses with inflammatory airway disease. J Vet Intern Med 31: 1193-1201, 2017.

36. Soiberman U, Kambhampati SP, Wu T, Mishra MK, Oh Y, Sharma R, Wang J, Al Towerki AE, Yiu S, Stark WJ and Kannan RM: Subconjunctival injectable dendrimer-dexamethasone gel for the treatment of corneal inflammation. Biomaterials 125: 38-53, 2017.

37. Kozan A, Kilic N, Alacam H, Guzel A, Guvenc T and Acikgoz M: The effects of dexamethasone and L-NAME on acute lung injury in rats with lung contusion. Inflammation 39: 1747-1756, 2016.

38. Liang J, Zhang B, Shen RW, Liu JB, Gao MH, Li Y, Li YY and Zhang W: Preventive effect of halofuginone on concanavalin A-induced liver fibrosis. PLoS One 8: e82232, 2013.

39. Carlson TJ, Pellerin A, Djuretic IM, Trivigno C, Koralov SB, Rao A and Sundrud MS: Halofuginone-induced amino acid starvation regulates Stat3-dependent Th17 effector function and reduces established autoimmune inflammation. J Immunol 192: 2167-2176, 2014.

40. Kotthoff P, Heine A, Held SAE and Brossart P: Dexamethasone induced inhibition of Dectin-1 activation of antigen presenting cells is mediated via STAT-3 and NF- $\kappa \mathrm{B}$ signaling pathways. Sci Rep 7: 4522, 2017

41. Yeh YC, Yang CP, Lee SS, Horng CT, Chen HY, Cho TH, Yang ML, Lee CY, Li MC and Kuan YH: Acute lung injury induced by lipopolysaccharide is inhibited by wogonin in mice via reduction of Akt phosphorylation and RhoA activation. J Pharm Pharmacol 68: 257-263, 2016.

42. Lu X, Pu Y, Kong W, Tang X, Zhou J, Gou H, Song X, Zhou H, Gao $\mathrm{N}$ and Shen J: Antidesmone, a unique tetrahydroquinoline alkaloid, prevents acute lung injury via regulating MAPK and NF- $\kappa \mathrm{B}$ activities. Int Immunopharmacol 45: 34-42, 2017.

43. He J, Zhou J, Yang W, Zhou Q, Liang X, Pang X, Li J, Pan F and Liang H: Dexamethasone affects cell growth/apoptosis/chemosensitivity of colon cancer via glucocorticoid receptor $\alpha / \mathrm{NF}-\kappa \mathrm{B}$. Oncotarget 8: 67670-67683, 2017.

44. Xu J, Liu D, Yin Q and Guo L: Tetrandrine suppresses $\beta$-glucan-induced macrophage activation via inhibiting NF- $\kappa B$ ERK and STAT3 signaling pathways. Mol Med Rep 13: $5177-5184,2016$
45. Huang CS, Lin AH, Yang TC, Liu KL, Chen HW and Lii CK: Shikonin inhibits oxidized LDL-induced monocyte adhesion by suppressing $\mathrm{NF} \kappa \mathrm{B}$ activation via up-regulation of PI3K/Akt/Nrf2-dependent antioxidation in EA.hy926 endothelial cells. Biochem Pharmacol 93: 352-361, 2015.

46. Li K, He Z, Wang X, Pineda M, Chen R, Liu H, Ma K, Shen H, Wu C, Huang N, et al: Apigenin C-glycosides of Microcos paniculata protects lipopolysaccharide induced apoptosis and inflammation in acute lung injury through TLR4 signaling pathway. Free Radic Biol Med 124: 163-175, 2018.

47. Qing R, Huang Z, Tang Y, Xiang Q and Yang F: Cordycepin alleviates lipopolysaccharide-induced acute lung injury via Nrf2/HO-1 pathway. Int Immunopharmacol 60: 18-25, 2018.

48. Chen LJ, Ding YB, Ma PL, Jiang SH, Li KZ, Li AZ, Li MC, Shi CX, Du J and Zhou HD: The protective effect of lidocaine on lipopolysaccharide-induced acute lung injury in rats through NF- $\mathrm{BB}$ and p38 MAPK signaling pathway and excessive inflammatory responses. Eur Rev Med Pharmacol Sci 22: 2099-2108, 2018.

49. Yavas G, Calik M, Calik G, Yavas C, Ata O and Esme H: The effect of Halofuginone in the amelioration of radiation induced-lung fibrosis. Med Hypotheses 80: 357-359, 2013.

50. Nagler A, Firman N, Feferman R, Cotev S, Pines M and Shoshan S: Reduction in pulmonary fibrosis in vivo by halofuginone. Am J Respir Crit Care Med 154: 1082-1086, 1996.

51. Wigenstam E, Elfsmark L, Ågren L, Akfur C, Bucht A and Jonasson S: Anti-inflammatory and anti-fibrotic treatment in a rodent model of acute lung injury induced by sulfur dioxide. Clin Toxicol (Phila) 56: 1185-1194, 2018.

52. Xu X, Zhu Q, Niu F, Zhang R, Wang Y, Wang W, Sun D, Wang X and Wang A: A2BAR activation attenuates acute lung injury by inhibiting alveolar epithelial cell apoptosis both in vivo and in vitro. Am J Physiol Cell Physiol 315: C558-C570, 2018.

53. Ehrentraut H, Weisheit C, Scheck M, Frede S and Hilbert T: Experimental murine acute lung injury induces increase of pulmonary TIE2-expressing macrophages. J Inflamm (Lond) 15: $12,2018$.

54. Dong SA, Zhang Y, Yu JB, Li XY, Gong LR, Shi J and Kang YY: Carbon monoxide attenuates lipopolysaccharide-induced lung injury by mitofusin proteins via p38 MAPK pathway. J Surg Res 228: 201-210, 2018

55. Takaoka Y, Goto S, Nakano T, Tseng HP, Yang SM, Kawamoto S, Ono K and Chen CL: Glyceraldehyde-3-phosphate dehydrogenase (GAPDH) prevents lipopolysaccharide (LPS)-induced, sepsis-related severe acute lung injury in mice. Sci Rep 4: 5204, 2014.

56. Yang Q, Liu X, Yao Z, Mao S, Wei Q and Chang Y: Penehyclidine hydrochloride inhibits the release of high-mobility group box 1 in lipopolysaccharide-activated RAW264.7 cells and cecal ligation and puncture-induced septic mice. J Surg Res 186: 310-317, 2014. 